\title{
Theoretical Model of a Nematogen: Estimation of Phase Stability, Absorption, Electrochemical, and Nonlinear Optical Properties
}

\author{
T. Jaison Jose ${ }^{a}$, A. Simi ${ }^{b}$, M. David RAJu ${ }^{c}$ And P. LAKshmi Praveen ${ }^{d, *}$ \\ ${ }^{a}$ P.G. Department of Chemistry, Andhra Loyola College, Vijayawada-520 008, Andhra Pradesh, India \\ ${ }^{b}$ Department of Chemistry, St. Joseph's College, Tiruchirapalli-620002, Tamil Nadu, India \\ ${ }^{c}$ Department of Chemistry, P.B. Siddhartha College of Arts \& Sciences, Vijayawada-520 010, Andhra Pradesh, India \\ ${ }^{d}$ Department of Physics, Veer Surendra Sai University of Technology, Burla-768018, Sambalpur, Odisha, India \\ (Received November 21, 2017; in final form May 7, 2018)

\begin{abstract}
Theoretical analysis has been presented on a nematogen 4-dimethylaminobenzaldehyde (4-cyanophenylethylidene) hydrazone $(\mathrm{E}, \mathrm{E})\left(\mathrm{C}_{18} \mathrm{H}_{18} \mathrm{~N}_{4}\right)$ to analyze its phase stability. The net atomic charges and dipole moment at each atomic centre has been calculated using complete neglect of differential overlap/spectroscopy method. The modified Rayleigh-Schrödinger perturbation theory along with multicentered-multipole expansion method has been used to evaluate the long-range intermolecular interactions. A "6-exp" potential function has been used for short-range interactions. The total interaction energy values have been used as input to calculate the probability of a particular configuration using the Maxwell-Boltzmann formula. Further, the Helmholtz free energy, and entropy at room temperature $(300 \mathrm{~K})$, nematic-isotropic transition temperature $(436 \mathrm{~K})$ and above transition temperature $(500 \mathrm{~K})$ have been computed. An attempt has been made to understand the phase stability and behaviour of the molecule. UV absorption spectra have been calculated using complete neglect of differential overlap/spectroscopy, and intermediate neglect of differential overlap/spectroscopy methods. The observed $\pi \rightarrow \pi^{*}$ transitions, electrochemical properties based on highest occupied molecular orbital, lowest unoccupied molecular orbital energies, and principal polarizability components, and anisotropy of polarizability have been reported to understand the kinetic stability, global reactivity, and non-linear optical activity of the molecule.
\end{abstract}

DOI: 10.12693/APhysPolA.134.512

PACS/topics: phase stability, UV absorption, reactivity, nematogen

\section{Introduction}

Number of mesophases will be observed during a transition from an ordered crystal to the disordered isotropic liquid, which are known as liquid crystalline (LC) phases [1]. These phases are with partial order of the crystalline state and certain degree of mobility. The quest of researchers to explicate the phase structure-ultra violet (UV) stability relationship at molecular level is the progressing theme of LC science $[2,3]$. From the LC literature, one may conclude that there are different kinds of nematic phase [4] among which the local molecular organization is qualitatively different. The research of particular nematogens demands a thorough knowledge of their structure in relation to that of other nematic phases based on theoretical models $[5,6]$ prior to the synthesis as it offers valuable information on phase behaviour. The stability of a given phase is principally governed by the Helmholtz free energy $A$. Molecular motions (translational, rotational etc.) have a direct effect on the entropy of a substance, since, the greater the energy that is stored in these motions, greater the degrees of freedom, and greater the entropy. Hence, the free energy is altered.

*corresponding author; e-mail: plpraveen_phy@vssut.ac.in
In general, the intramolecular electronic energy is much higher than the intermolecular potential energy, a phase transition results in a very small change in the electronic energy of a molecule. The molecular structure remains unchanged despite the phase transitions in such cases.

The phase structure arises from a delicate interplay between the internal molecular structure and the moleculemolecule interaction energy, which has a close relation with anisotropy of physical properties [7]. These mesophases are controlled by the interplay between probability and entropy. Further, the analysis of the electronic excited states of mesogenic molecules may also have some interest for the study of LC behaviour. In fact, the orientation-dependent part of the dispersive intermolecular forces, which also contribute to the stabilization of the LC phase, particularly of the long range orientational order of nematics, depends on the spatial anisotropy of molecular transition moments from fundamental to excited electronic states (molecular anisotropy factor) [8].

The role of molecular interactions in LC compounds has engrossed the attention of several workers $[9,10]$ based on the Rayleigh-Schrödinger perturbation method. These studies have been focused at establishing the anisotropic nature of the pair potential, and subsequently finding out the maximum probable configuration of a pair of LC molecules. Thus, the main emphasis was laid on 
finding out the minimum energy with observed crystal structure, the basic motive for ordering of a molecule. It has been observed that the interaction energies for a pair of mesogens indicate the preference of a particular configuration over the other depending on their energy values. These values, however, do not reflect the actual relative preference, which can only obtain through their probability corresponding to each configuration. Further, in terms of multipole interactions, significant differences among the energies of various configurations are noticed, which must have a marked effect on thermodynamic properties of the system. Hence, the relative energies are used as an input to calculate the thermodynamic parameters of $\mathrm{C}_{18} \mathrm{H}_{18} \mathrm{~N}_{4}$.

The relative energies (the difference of energy at a particular transition/rotation point to the minimum energy of a configuration) between a molecular pair of $\mathrm{C}_{18} \mathrm{H}_{18} \mathrm{~N}_{4}$ have been computed at an intermediate distance of $6 \AA$ for stacking and $8 \AA$ for in-plane interactions. Similarly, a distance of $20 \AA$ has been kept for terminal interactions. The selection of intermolecular separations has been made to allow the molecule to have full freedom corresponding to rotation and translation relative to each other. Furthermore, instead of finding the exact minimum energy configuration an attempt has been made to elucidate the general behavior of the molecules surrounding a fixed molecule in a particular frame of reference. An examination of thermodynamic data has revealed that $\mathrm{C}_{18} \mathrm{H}_{18} \mathrm{~N}_{4}$ exhibits nematic-isotropic transition temperature at $436 \mathrm{~K}$ [11]. Further, the semiempirical analysis aims at providing a picture of molecular absorption, electrochemical, and nonlinear optical properties of $\mathrm{C}_{18} \mathrm{H}_{18} \mathrm{~N}_{4}$ using the complete neglect of differential overlap/spectroscopy $(\mathrm{CNDO} / \mathrm{S})[12,13]$, intermediate neglect of differential overlap/spectroscopy (INDO/S) [14, 15] schemes. Global chemical reactivity descriptors are reported to understand stability and reactivity, while polarizability components are analyzed to understand the nonlinear optical activity.

\section{Theoretical approach}

The molecular geometry of $\mathrm{C}_{18} \mathrm{H}_{18} \mathrm{~N}_{4}$ has been constructed on the basis of published crystallographic data with the standard values of bond lengths and bond angles [11]. The details of the computations carried out are presented in the next subsections.

\subsection{Computation of atomic net charges and dipole moments}

The simplified formula for interaction energy calculations requires the evaluation of atomic net charges and dipole moment components at each atomic centre through an all-valence electron method. In the present computation, the CNDO/S method [16] has been employed to compute the net atomic charges and dipole moment at each atomic centre of the molecule.

\subsection{Computation of configurational probabilities}

According to the second order perturbation theory for intermediate range interactions [17], the total pair interaction energy of molecules $\left(U_{\text {pair }}\right)$ is represented as sum of various terms contributing to the total energy

$$
U_{\text {pair }}=U_{e l}+U_{p o l}+U_{d i s p}+U_{r e p},
$$

where $U_{e l}, U_{p o l}, U_{d i s p}$ and $U_{r e p}$ are the electrostatic, polarization, dispersion, and repulsion energy terms, respectively. The electrostatic term is expressed as

$$
U_{e l}=U_{Q Q}+U_{Q M I}+U_{M I M I}+\ldots,
$$

where $U_{Q Q}, U_{Q M I}$ and $U_{M I M I}$ etc. are monopolemonopole, monopole-dipole and dipole-dipole terms, respectively. In fact, the inclusion of higher order multipoles does not affect significantly the electrostatic interaction energy and the calculation only up to dipoledipole term gives satisfactory result [17-19]. The dispersion and short-range repulsion terms are considered together because several semi-empirical approaches, viz. the Lennard-Jones or Buckingham type approach, actually proceed in this way. The necessary formulae may be found elsewhere [19]. An orthogonal coordinate system is considered to facilitate the above calculation. The origin has been chosen at almost centre of the molecule. The $x$-axis along a bond parallel to the long molecular axis while the $y$-axis lies in the plane of the molecule and $z$-axis perpendicular to the molecular plane.

The total interaction energy values obtained through these computations were used as input to calculate the probability of occurrence of a particular configuration $i \quad\left(P_{i}\right)$ using the Maxwell-Boltzmann formula [19] in order to obtain a better insight

$$
P_{i}=\frac{\mathrm{e}^{-\Delta \varepsilon_{i} / k T}}{\sum_{j} \mathrm{e}^{-\Delta \varepsilon_{j} / k T}} .
$$

\subsection{Computation of thermodynamic parameters}

The following thermodynamic parameters [20] have been calculated to understand the phase stability and phase behaviour at molecular level:

$$
\begin{aligned}
& A=-k T \ln \sum_{i} \exp \left(-\beta \varepsilon_{i}\right) \\
& S=k \ln \sum_{i} \exp \left(-\beta \varepsilon_{i}\right)+(U / T),
\end{aligned}
$$

where the internal energy $U$ is expressed as

$$
U=\sum_{i} \varepsilon_{i} \exp \left(-\beta \varepsilon_{i}\right) / \sum_{i} \exp \left(-\beta \varepsilon_{i}\right)
$$

where $P_{i}$ stands for probability, $A$ stands for the Helmholtz free energy, and $S$ stands for entropy, $\Delta \varepsilon_{i}$ is the relative energy, $\beta=1 / k T, k$ is the Boltzmann constant, $T$ is the absolute temperature and $\varepsilon_{i}$ represents the energy of the configuration $i$ to the minimum energy value in a particular set for which the probability distribution is computed. 


\subsection{UV absorption spectra}

The present study adopts two methods for the estimation of UV spectra of $\mathrm{C}_{18} \mathrm{H}_{18} \mathrm{~N}_{4}$ molecule namely $\mathrm{CNDO} / \mathrm{S}$, and INDO/S. Revised versions of QCPE 174 by Reimers, University of Sydney and co-workers have been used for the semiempirical calculations [21]. Global and local reactivity descriptors from the Koopman theorem, the ionization potential IP and electron affinity EA are the eigenvalue of the highest occupied molecular orbital (HOMO) and lowest unoccupied molecular orbital (LUMO) with change of sign [22] i.e., IP $\approx E_{H}$ and EA $\approx E_{L}$. Several GCRD parameters of molecules such as hardness $\eta$, chemical potential $\mu$, softness $S$, electronegativity $\chi$ and electrophilicity index $\omega$ have been calculated based on the basis of CNDO/S, and INDO/S methods. The global hardness $\eta$, and chemical potential $\mu[23]$ is defined as the second and first derivative of the energy $E$, with respect to the number of electrons $N$, at constant external potential, $v(r)$, captures the resistance of a chemical species to changing its electronic number

$$
\begin{aligned}
\mu & =\left(\frac{\partial E}{\partial N}\right)_{v} \approx-\frac{\varepsilon_{\mathrm{HOMO}}+\varepsilon_{\mathrm{LUMO}}}{2} \\
\eta & =\frac{1}{2}\left(\frac{\partial^{2} E}{\partial N^{2}}\right)_{v} \approx \frac{\varepsilon_{\mathrm{LUMO}}-\varepsilon_{\mathrm{HOMO}}}{2} m \\
\chi & =-\mu,
\end{aligned}
$$

where $E$ and $v(r)$ are electronic energy and external potential of an $N$-electron system, respectively. Softness [24] is a property that measures the extent of chemical reactivity. It is the reciprocal of hardness and electronegativity has been defined as the negative of the electronic chemical potential in the Mulliken sense

$$
\text { Softness }=\left(\frac{\partial N}{\partial \mu}\right)_{v}=\left(\frac{\partial \rho(r)}{\partial \mu}\right)_{v}=\frac{1}{\eta}
$$

Using the Koopman theorem for closed shell molecules $\eta, \mu$ and $\chi$ can be redefined as: $\chi=(I P+E A) / 2$, chemical hardness $\eta=(I P-E A) / 2$, electronic chemical potential $\mu=-(I P+E A) / 2$. The concept of electrophilicity viewed as a reactivity index was introduced by Lee et al. [25]. It is based on a second order expansion of the electronic energy with respect to the charge transfer $\Delta N$ at fixed geometry. This index, which measures the stabilization in energy when the system acquires an additional electronic charge $\Delta N$ from the environment, is defined by the following simple and more familiar form [26] in terms of the electronic chemical potential $\mu$ and the chemical hardness $\eta$. Electrophilicity is a useful structural descriptor of reactivity and is frequently used in the analysis of the chemical reactivity of molecules, which is expressed as: $\omega=\mu^{2} / \eta$. The inverse of the electrophilicity is defined as nucleophilicity and is expressed as: $v=1 / \omega$. On the other hand, the maximum amount of electronic charge that an electrophile system may accept is given by $\Delta N_{\max }=-\mu / \eta[23]$. The maximum charge transfer $\Delta N_{\max }$ toward the electrophile may be evaluated. Thus, while the quantity $\Delta N_{\max }$ describes the tendency of the system to acquire additional electronic charge from the environment; the quantity $\omega$ describes the charge capacity of the molecule.

\section{Results and discussion}

The molecular geometry of $\mathrm{C}_{18} \mathrm{H}_{18} \mathrm{~N}_{4}$ with various atoms is shown in Fig. 1. The results of probability distribution corresponding to the different modes of interactions are discussed below.

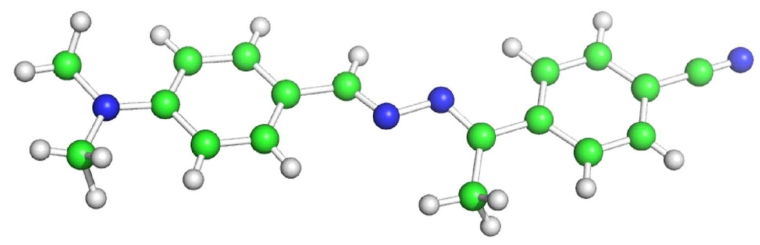

Fig. 1. Molecular geometry of $\mathrm{C}_{18} \mathrm{H}_{18} \mathrm{~N}_{4}$ along with various atoms (green - carbon, white - hydrogen, blue - hydrogen).

\subsection{Stacking interactions}

One of the interacting molecules is fixed in the $x-y$ plane, while the second has been kept at a separation of $6 \AA$ along the $z$-axis with respect to the fixed one. The variation of probability with respect to translation along $x$-axis corresponding to configuration $y\left(0^{\circ}\right) z\left(0^{\circ}\right)$ is shown in Fig. 2 at room temperature $(300 \mathrm{~K})$, nematic-

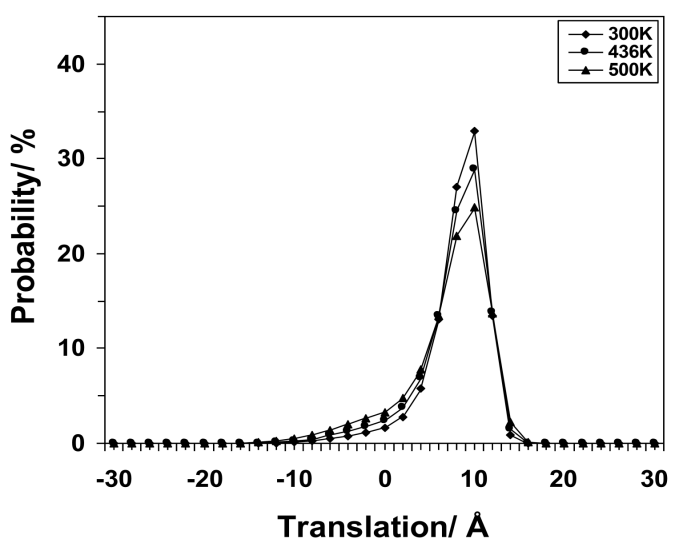

Fig. 2. Variation of probability with respect to translation along $x$-axis during stacking interactions at room temperature $300 \mathrm{~K}$, nematic-isotropic transition temperature $436 \mathrm{~K}$, and above phase transition temperature $500 \mathrm{~K}$.

isotropic transition temperature $(436 \mathrm{~K})$ and above phase transition temperature $(500 \mathrm{~K})$. It is clear that the variation of probability is constant in the region of $-10 \pm 2 \AA$, which shows that sliding of one molecule over the other is energetically allowed for a small range. This may be correlated with the fluidity of the compound maintaining its alignment in the mesophase. Further, the configuration 
has been refined with respect to translation along the $x$ axis at the equilibrium condition, the energy is brought down and the configurational probability is again investigated with respect to rotation about the $x$-axis.

The variation of probability with respect to rotation about $x$-axis corresponding to configuration $y\left(180^{\circ}\right)$ $z\left(0^{\circ}\right)$ has been carried out. This analysis indicates that the maximum probability shows a slight preference for the aligned structure of this configuration. The minimum energy thus obtained is taken as the starting point and the entire process is repeated for small intervals. The energy is minimized with respect to translations and rotations about $x, y$, and $z$-axes. An accuracy of $0.1 \AA$ in translation and $1^{\circ}$ in rotation of one molecule with respect to the other has been achieved.

\subsection{In-plane interactions}

During this mode, the interacting molecule has been kept at a separation of $8 \AA$ along the $y$-axis with respect to the fixed one. The effect of translation along $x$-axis corresponding to the configuration $y\left(0^{\circ}\right)$ is shown in Fig. 3. The probability has been computed at room temperature $(300 \mathrm{~K})$, nematic-isotropic transition temperature $(436 \mathrm{~K})$, and above phase transition temperature $(500 \mathrm{~K})$. Since in-plane interactions are weaker than the stacking interactions, a greater freedom corresponding to translation is observed with the maximum probability at $4 \AA$. The interacting configurations have been refined with respect to translation along the $x$-axis at the equilibrium condition, the energy is brought down and the probability is further investigated with respect to rotation about $x$-axis.

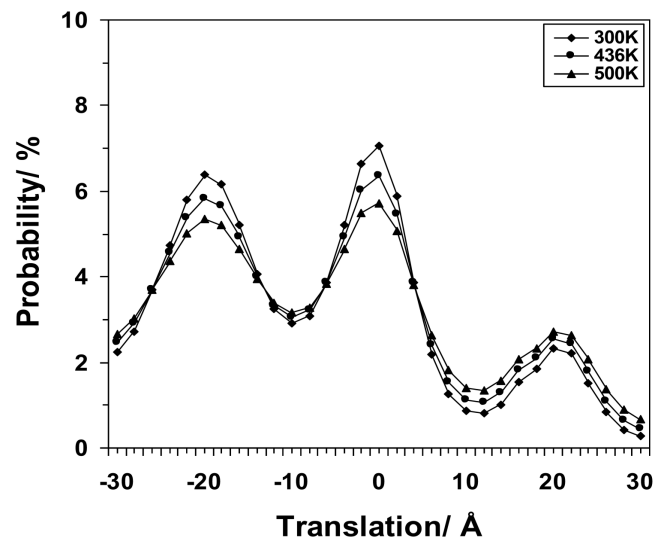

Fig. 3. As in Fig. 2, but for in-plane interactions.

The variation of probability with respect to rotation about the $x$-axis corresponding to configuration $y\left(180^{\circ}\right)$ has been carried out. It has been noticed that a pronounced peak exists for a particular rotation point, and all the remaining regions have negligible probability as compared to this configuration. The noteworthy aspect in this study is that the rotational freedom is much more pronounced as compared to the stacking interactions.
The variation of the probability with respect to rotation about the $y$-axis corresponding to the configuration $x\left(0^{\circ}\right)$ has also been carried out, and it is observed that the rotation about the $y$-axis does not alter the configurational probability to a great extent compared to rotation about $x$-axis.

\subsection{Terminal interactions}

These interactions are weakest but become important when the molecule possesses a polar group at either or both of the ends or if there is a possibility of hydrogen bonding. To investigate the terminal interactions apart from the van der Waals forces, the interacting molecule have been shifted along the $x$-axis by $20 \AA$ with respect to the fixed one, and rotations were allowed about $x$ axis. The rotations about the $x$-axis corresponding to configuration $y\left(0^{\circ}\right)$ show (Fig. 4) no preference for any angle, i.e., the molecules are completely free to rotate about their long molecular axis.

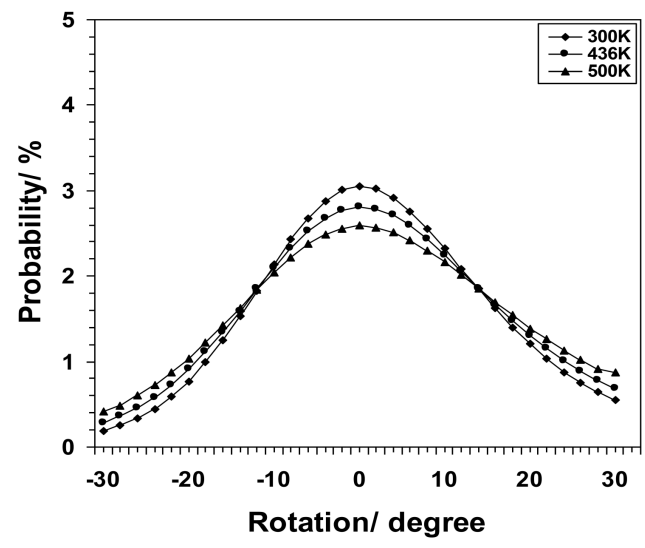

Fig. 4. Variation of probability with respect to rotation about $x$-axis during terminal interactions at room temperature $300 \mathrm{~K}$, nematic-isotropic transition temperature $436 \mathrm{~K}$, and above phase transition temperature $500 \mathrm{~K}$.

\section{Phase stability and phase behaviour}

The present analysis may be helpful to understand the phase stability well as nematic character of $\mathrm{C}_{18} \mathrm{H}_{18} \mathrm{~N}_{4}$. The nematic behaviour of LC is generally manifested by its translational freedom along the long molecular axis. The thermodynamic parameters such as the Helmholtz free energy and entropy have been calculated with respect to translation along $x$-axis during the different modes of interactions to estimate the phase stability and phase behaviour.

A comparative picture of the Helmholtz free energy with respect to translation and rotation during the different modes of interactions at room temperature $(300 \mathrm{~K})$, nematic-isotropic transition temperature (436 K), and above phase transition temperature $(500 \mathrm{~K})$ is listed in Table I. As the negative free energy increases, the 
phase stability of the system also increases. It is evident from Table I that the Helmholtz free energy during the stacking interactions at room temperature is $8.37 \mathrm{kcal} / \mathrm{mol}$, while it is increased to $-9.58 \mathrm{kcal} / \mathrm{mol}$ at nematic-isotropic transition temperature, which confirms the stability (order) of molecule in nematic phase. It is much more prominent during in-plane and terminal interactions as observed from Table I.

TABLE I

A comparative picture of thermodynamic parameter, Helmholtz free energy $(A)$ corresponding to various configurations during the different modes of interactions at room temperature $(300 \mathrm{~K})$, nematic-isotropic transition temperature $(436 \mathrm{~K})$, and above phase transition temperature $(500 \mathrm{~K})$.

\begin{tabular}{c|c|c|c|c}
\hline \hline \multirow{2}{*}{ Mode } & \multirow{2}{*}{ Configuration } & \multicolumn{3}{|c}{$A\left[\mathrm{kcalmol}^{-1}\right]$} \\
\cline { 3 - 5 } & & $300 \mathrm{~K}$ & $436 \mathrm{~K}$ & $500 \mathrm{~K}$ \\
\hline stacking $^{a}$ & $y\left(0^{\circ}\right) z\left(0^{\circ}\right)$ & -8.37 & -9.58 & -10.45 \\
stacking $^{b}$ & $y\left(180^{\circ}\right) z\left(0^{\circ}\right)$ & -5.13 & -5.99 & -7.21 \\
in-plane $^{a}$ & $y\left(0^{\circ}\right)$ & -4.43 & -5.55 & -6.69 \\
in-plane $^{b}$ & $y\left(180^{\circ}\right)$ & -4.58 & -4.86 & -6.75 \\
terminal $^{b}$ & $y\left(0^{\circ}\right)$ & -3.13 & -4.84 & -5.05 \\
\hline
\end{tabular}

${ }^{a}$ translation along $x$-axis, ${ }^{b}$ rotation about $y$-axis

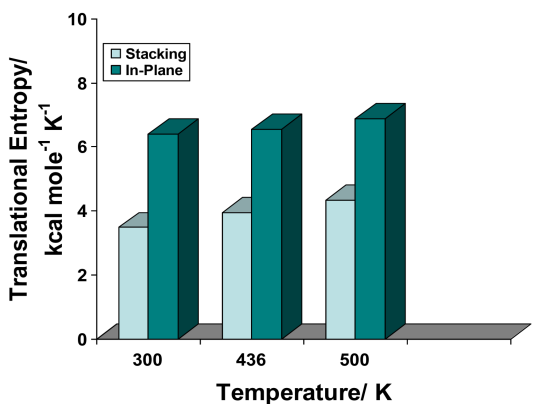

Fig. 5. Translational entropy as a function of temperature during stacking and in-plane interactions at room temperature $300 \mathrm{~K}$, nematic-isotropic transition temperature $436 \mathrm{~K}$ and above phase transition temperature $500 \mathrm{~K}$.

Figure 5 shows the translational entropy as a function of temperature during stacking and in-plane interactions along the long molecular axis. It is noticed that translational entropy during stacking interactions is $3.95 \mathrm{kcal} \mathrm{mol}^{-1} \mathrm{~K}^{-1}$ at nematic-isotropic transition temperature $(436 \mathrm{~K})$. However, at room temperature $\left(300 \mathrm{~K}\right.$ ) the value is $3.49 \mathrm{kcal} \mathrm{mol}^{-1} \mathrm{~K}^{-1}$ (with a $\Delta S$ of $0.46 \mathrm{kcal} \mathrm{mol}^{-1} \mathrm{~K}^{-1}$ ) indicating a strong binding at low temperature with less disorder. But with increase of temperature, the molecules obtain sufficient freedom to slide along the long molecular axis, which causes the increment of disorder. Such translational freedom is much more dominant during in-plane mode. Evidently, even at room temperature the value is $6.41 \mathrm{kcal} \mathrm{mol}^{-1} \mathrm{~K}^{-1}$, which increased to $6.55 \mathrm{kcal} \mathrm{mol} \mathrm{K}^{-1}$ (with a $\Delta S$ of $0.14 \mathrm{kcal} \mathrm{mol}^{-1} \mathrm{~K}^{-1}$ ) at nematic-isotropic transition temperature $(436 \mathrm{~K})$. It may be noted that though the freedom is considerable for smaller translation, longer translations are not generally permitted. Thus, in the mesomorphic range, small movements of molecules are only possible. During a phase transition, the molecular motions would be excited and thereby the number of thermally accessible conformations would be increased. The translational entropy in the isotropic liquid states is increased from its room temperature (Fig. 5). It implies that the different modes of molecular motions (translational, rotational, etc.) are excited almost to an equal extent. This induces all possible degrees of freedom, and leads to the isotropic state. The comparison of translational entropy during stacking and in-plane interactions indicates a low value of $\Delta S$ during in-plane interactions, which indicates the higher ordering along $y$-axis. This shows that the molecule $\mathrm{C}_{18} \mathrm{H}_{18} \mathrm{~N}_{4}$ does not show any preference in forming the stacked layers (along $z$-axis), hence justifies the nematic character.

\section{UV absorption spectrum}

In the calculation of electronic spectra, the configuration interaction (CI) method is widely employed. Using a CI method in combination with a semi-empirical model Hamiltonian, an evaluation of absorption spectra of large organic molecules and LCs becomes possible [27]. The analysis of UV-Vis absorption spectra of $\mathrm{C}_{18} \mathrm{H}_{18} \mathrm{~N}_{4}$ molecule has been given below using $\mathrm{CNDO} / \mathrm{S}$ and INDO $/ \mathrm{S}$ methods.

\section{1. $C N D O / S$ and $I N D O / S$ data}

The UV absorption spectrum of $\mathrm{C}_{18} \mathrm{H}_{18} \mathrm{~N}_{4}$ molecule has been shown in Fig. 6 using CNDO $/ \mathrm{S}$ and INDO/S methods. A three-band structure has been observed using $\mathrm{CNDO} / \mathrm{S}$ method in the UV region with absorptions at $209.37 \mathrm{~nm}\left(\lambda_{1}\right), 273.24 \mathrm{~nm}\left(\lambda_{2}\right)$, and $311.33 \mathrm{~nm}\left(\lambda_{3}\right)$. The INDO/S method estimates four strong absorptions at $214.65 \mathrm{~nm}\left(\lambda_{1}\right), 239.26 \mathrm{~nm}\left(\lambda_{2}\right), 291.99 \mathrm{~nm}\left(\lambda_{3}\right)$, and $321.29 \mathrm{~nm}\left(\lambda_{4}\right)$. The $\lambda_{\max }$ (which provides UV stability data of the compound) has been observed at $\lambda_{3}$ and $\lambda_{4}$ using $\mathrm{CNDO} / \mathrm{S}$ and INDO/S methods, respectively. These bands arise from the HOMO $\rightarrow$ LUMO transition, and it is assigned as $\pi \rightarrow \pi^{*}$ transition in the molecule. The other absorption bands corresponding to the remaining wavelengths in UV region also indicate the possibility of $\pi \rightarrow \pi^{*}$ transitions in the molecule at higher wavelengths. In particular, it is found that the transition corresponding to the most intense peak in the UV spectrum of the molecules ( $\pi \rightarrow \pi^{*}$ transition) has a strong polarization along the long molecular axis. The absorption maxima $\left(\lambda_{\max }\right)$, extinction coefficients, oscillator strength $f$, vertical excitation energies $E_{V}$, dipole moment $\mu$ and its components $\left(\mu_{x}, \mu_{y}, \mu_{z}\right)$, HOMO $(H)$, LUMO $(L)$, and energy gap $E_{g}$ of $\mathrm{C}_{18} \mathrm{H}_{18} \mathrm{~N}_{4}$ molecule, using the $\mathrm{CNDO} / \mathrm{S}$ and INDO/S methods, has been reported in Table II. It is evident from the table that both 
the methods estimate the values of $\lambda_{\max }$, energy gap, and other parameters up to a reasonable agreement. The chemical hardness, potential, reactivity, softness, electronegativity, electrophilicity, kinetic stability and optical polarizability, etc. of any molecule can be explained with the help of $\mathrm{H}$ and $\mathrm{L}$ energies. The packing density of $\mathrm{C}_{18} \mathrm{H}_{18} \mathrm{~N}_{4}$ is expected to be high due to the planarity of the molecule. This induces charge transfer that is responsible for the nonlinear optical properties.

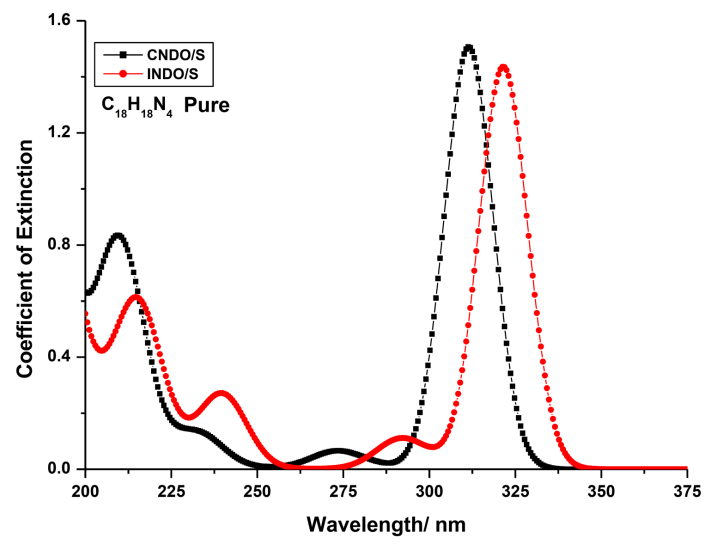

Fig. 6. UV absorption spectra of $\mathrm{C}_{18} \mathrm{H}_{18} \mathrm{~N}_{4}$ molecule using $\mathrm{CNDO} / \mathrm{S}$ and INDO/S methods. Extinction unit: $10^{4} \mathrm{dm}^{3} \mathrm{~mol}^{-1} \mathrm{~cm}^{-1}$.

TABLE II

The absorption bands and maxima $\lambda_{\max }\left(^{*}\right)[\mathrm{nm}]$, extinction coefficient $n\left[10^{4} \mathrm{dm}^{3} \mathrm{~mol}^{-1} \mathrm{~cm}^{-1}\right]$, oscillator strength $f$, vertical excitation energies $E_{V}$, dipole moment $\mu[\mathrm{D}]$ and its components $\mu_{x}, \mu_{y}, \mu_{z}, \operatorname{HOMO}(H)$, LUMO $(L)$, and energy gap $\left(E_{g}\right)$ of $\mathrm{C}_{18} \mathrm{H}_{18} \mathrm{~N}_{4}$ molecule using the $\mathrm{CNDO} / \mathrm{S}$ and INDO/S methods.

\begin{tabular}{c|c|c|c|c|c|c|c}
\hline \hline$\lambda_{\max }$ & $n$ & $E_{V}$ & $f$ & $\mu_{x}$ & $\mu_{y}$ & $\mu_{z}$ & $\mu$ \\
\hline $\mathrm{CNDO} / \mathrm{S}, H=-8.48 \mathrm{eV}, L=-1.83 \mathrm{eV}, E_{g}=6.65 \mathrm{eV}$ \\
\hline 209.37 & 0.83 & 5.93 & 0.64 & 1.07 & 1.79 & 13.75 & 13.91 \\
273.24 & 0.06 & 4.55 & 0.05 & 0.62 & 2.61 & 7.28 & 7.76 \\
$311.33^{*}$ & 1.51 & 3.98 & 1.51 & 0.87 & 2.09 & 14.33 & 14.51 \\
\hline \\
\hline
\end{tabular}

\subsection{Electrochemical and non-linear optical properties}

Global chemical reactivity descriptors (GCRD) are used to understand the relationship between global chemical reactivity, structure, stability etc. parameters of any molecule. The hardness of any molecule is related to aromaticity [28]. The values of various GCRD parameters such as chemical hardness $\eta$, chemical potential $\mu$, softness $S$, electronegativity $\chi$ and electrophilicity index $\omega$ of the molecules using HOMO and LUMO energies have been given in Table III. The calculated $\mathrm{H}-\mathrm{L}$ energy gap and chemical hardness values reveal the chemical stability of titled molecules. The higher energy gap suggests higher kinetic stability as according to softness-hardness rule. When the molecule possesses wide energy gap then is said to be hard and also shows higher thermal and kinetic stability. The smaller $\mathrm{H}-\mathrm{L}$ energy gap implies lower kinetic stability and higher chemical reactivity because it is energetically favorable to add electrons to a high lying LUMO or to extract electrons from a low lying HOMO.

\section{TABLE III}

Calculated values of ionization energy $I=\left(-E_{H}\right)$, electron affinity $A=\left(-E_{L}\right)$, electronegativity $\chi=(I+A) / 2$, chemical hardness $\eta=(I-A) / 2$, electronic chemical potential $\mu=-(I+A) / 2$, electrophilicity index $\omega=\mu^{2} / \eta$, and softness $S=1 / \eta$, maximum charge transfer $\Delta N_{\max }$, principal polarizability components $\alpha_{X X}, \alpha_{Y Y}, \alpha_{Z Z}$ in a.u., isotropic component $\alpha^{i s o}$ ), anisotropy $\Delta \alpha$, asymmetry $\eta_{\alpha}$, and $\Delta \alpha / \alpha$ of $\mathrm{C}_{18} \mathrm{H}_{18} \mathrm{~N}_{4}$ molecule using $\mathrm{CNDO} / \mathrm{S}$ and INDO/S methods.

\begin{tabular}{c|c|c}
\hline \hline Parameter & CNDO/S method & INDO/S method \\
\hline$I[\mathrm{eV}]$ & 8.48 & 7.05 \\
$A[\mathrm{eV}]$ & 1.83 & 0.69 \\
$\chi[\mathrm{eV}]$ & 5.15 & 3.87 \\
$\eta[\mathrm{eV}]$ & 3.32 & 3.18 \\
$\mu[\mathrm{eV}]$ & -5.15 & -3.87 \\
$\omega[\mathrm{eV}]$ & 7.98 & 4.71 \\
$S\left[\mathrm{eV}{ }^{-1}\right]$ & 0.30 & 0.31 \\
$\alpha_{X X}$ & 1.63 & 2.76 \\
$\alpha_{Y Y}$ & 116.17 & 116.47 \\
$\alpha_{Z Z}$ & 310.05 & 324.89 \\
$\alpha^{i s o}$ & 142.61 & 148.04 \\
$\Delta \alpha$ & 211.48 & 217.92 \\
$\eta_{\alpha}$ & 1.37 & 1.43 \\
$\Delta \alpha / \alpha$ & 1.48 & 1.47
\end{tabular}

Polarizability values of molecules are very important in investigating electrostatic intermolecular interaction energies of molecules without having permanent dipole moment. Polarizability is the measure of distortion of a molecule in an electric field. The quantities $\alpha_{x x}, \alpha_{y y}$ and $\alpha_{z z}$ are known as the principal values of the polarizability tensor. This property measures the strength of molecular interactions (e.g., long-range intermolecular induction, dispersion forces, etc.), and the optical properties of a system. The calculated principal polarizability components $\left(\alpha_{x x}, \alpha_{y y}, \alpha_{z z}\right)$, isotropic component $\left(\alpha^{i s o}\right)=\left(\alpha_{x x}+\alpha_{y y}+\alpha_{z z}\right) / 3$, anisotropy $\Delta \alpha={ }_{\mid} \alpha_{x x^{-}}$ $\left(\alpha_{y y}+\alpha_{z z}\right) / 2$, asymmetry $\eta_{\alpha}=\left(\alpha_{y y}-\alpha_{z z} / \alpha_{x x}-\alpha^{i s o}\right)$ of $\mathrm{C}_{18} \mathrm{H}_{18} \mathrm{~N}_{4}$ has been presented in Table IV. The molecular polarizability component $\alpha_{z z}$ is higher than the other two polarizability components. The ratio $\Delta \alpha / \alpha$ is directly related to the strength of the anisotropic dispersion energy for the optimized geometry of the molecules. The need of experimental data on polarizability of $\mathrm{C}_{18} \mathrm{H}_{18} \mathrm{~N}_{4}$ put a stop to full confidence being given in the data of Table III. Also, accurate theoretical calculations of molecu- 
lar polarizabilities are rather difficult to obtain with more complex methods. Hence, it may be concluded that the data in Table III have significance at a comparative level. Further, it should also be noted that this data refers to "gas phase" molecule. A decrement in these values is to be expected when the dielectric nature of the material is considered. With this limitation, too, the data and the geometric data discussed above are consistent with the LC behaviour of $\mathrm{C}_{18} \mathrm{H}_{18} \mathrm{~N}_{4}$.

\section{Conclusions}

The present theoretical model leads to the following conclusions:

1. The comparison of translational entropy during stacking and in-plane interactions indicates a low value of $\Delta S$ during in-plane interactions, which indicates the higher ordering along $y$-axis. This shows that the molecule $\mathrm{C}_{18} \mathrm{H}_{18} \mathrm{~N}_{4}$ does not show any preference in forming the stacked layers (along $z$-axis), hence justifies the nematic character. The increment in negative free energy confirms the stability (order) of molecule in nematic phase.

2. It is evident that both the methods estimate the values of $\lambda_{\max }$, energy gap, and other parameters up to a reasonable agreement. The packing density of $\mathrm{C}_{18} \mathrm{H}_{18} \mathrm{~N}_{4}$ is expected to be high due to the planarity of the molecule. This induces charge transfer that is responsible for the nonlinear optical properties.

3. The ratio $\Delta \alpha / \alpha$ is directly related to the strength of the anisotropic dispersion energy for the optimized geometry of the molecules. For the title compound it is $>1$ using both the methods.

\section{References}

[1] S. Chandrasekhar, Liquid Crystals, Cambridge University Press, 1992.

[2] V.G. Chigrinov, V.M. Kozenkov, H.S. Kwok, Photoalignment of Liquid Crystalline Materials: Physics and Applications, Wiley, England 2008.

[3] R. Chen, Z. An, W. Wang, X. Chen, P. Chen, Liq. Cryst. 44, 1515 (2017).
[4] A. De Vries, Mol. Cryst. Liq. Cryst. 10, 31 (1970).

[5] P. Das, P. Lakshmi Praveen, Mol. Cryst. Liq. Cryst. 652, 185 (2017).

[6] K.P. Sokolowsky, H.E. Bailey, M.D. Fayer, J. Phys. Chem. B 118, 7856 (2014).

[7] B. Kamala Latha, K.P.N. Murthy, V.S.S. Sastry, Phys. Rev. E 96, 032703 (2017).

[8] J.W. Goodby, Liq. Cryst. 44, 1755 (2017).

[9] L.H.M. Huizar, C.H.R. Reyes, E.G. Sanchez, J. Mol. Liq. 199, 530 (2014).

[10] R.K. Srivastav, M. Roychoudhury, J. Kumar, D. Kumar, Mol. Cryst. Liq. Cryst. 652, 51 (2017).

[11] R. Centore, C. Garzillo, J. Chem. Soc. Perkin Trans. 2, 79 (1997).

[12] T. Bouman, D.A. Lightner, J. Am. Chem. Soc. 98, 3145 (1979)

[13] A.P. Volosov, V.A. Zublov, Theor. Chim. Acta 44 375 (1977).

[14] H. Komori, Y. Inai, J. Phys. Chem. A 110, 9099 (2006).

[15] S.I. Kawmura, T. Morita, S. Kimura, Sci. Technol. Adv. Mater. 7, 544 (2006).

[16] P.E. Stevenson, J. Phys. Chem. 76, 2424 (1972).

[17] V. Tomar, T.F. Roberts, N.L. Abbott, J.P. Hernández-Ortiz, J.J. de Pablo, Langumir 28, 6124 (2012).

[18] D.P. Ojha, P. Lakshmi Praveen, J. Phys. Chem. Solids 74, 1653 (2013).

[19] P. Lakshmi Praveen, D.P. Ojha, Mol. Cryst. Liq. Cryst. 608, 72 (2015).

[20] J.O. Hirschfelder, C.F. Curtiss, R.B. Bird, Molecular Theory of Gases and Liquids, Wiley, USA 1967.

[21] B. Tejerina, J. Reimers, CNDO/INDO, 2014.

[22] R.T. Myers, J. Inorg. Nuclear Chem. 43, 3083 (1981).

[23] R.G. Parr, R.A. Donnelly, M. Levy, W.E. Palke, J. Chem. Phys. 68, 3801 (1978).

[24] W. Yang, R.G. Parr, Proc. Natl. Acad. Sci. USA 82 , $6723(1985)$

[25] C. Lee, W. Yang, G.R. Parr, Phys. Rev. B 37, 785 (1988).

[26] R.G. Parr, L.V. Szentpály, S. Liu, J. Am. Chem. Soc. 121, 1922 (1999).

[27] M.P. Crozet, P. Vanelle, L. Bouscasse, T. Avignon, Spectrosc. Lett. 19, 1049 (1986).

[28] R. Vijayaraj, P.K. Subramanian, P.K. Chattaraj, J. Chem. Theor. Comput. 5, 2744 (2009). 